\title{
Solvent Free Microwave Accelerated Synthesis of Heterocyclic Thiazolidin-4-ones as Antimicrobial and Antifungal Agents
}

\author{
Kondapalli Venkata Gowri Chandra Sekhar, ${ }^{\dagger, *}$ Vajja Sambasiva Rao, ${ }^{\dagger}$ Aravalli Satish Reddy, Ravada Sunandini, \\ and V S A Kumar Satuluri
}

\author{
Chemistry Group, BITS, Pilani-333 031, Rajasthan, India \\ ${ }^{\dagger}$ Chemistry Group, BITS, Pilani-Hyderabad Campus, Jawahar Nagar, Shamirpet Mandal, Ranga Reddy District, \\ Hyderabad-500 078, Andhra Pradesh, India. "E-mail: kvgc@bits-hyderabad.ac.in \\ Received January 4, 2010, Accepted March 3, 2010
}

\begin{abstract}
A simple and efficient method has been developed for conversion of arenecarbaldehyde-3-methylquinoxalin-2-ylhydrazones to 3-(2-methylquinoxalin-3-yl)-2-(substitutedphenyl)thiazolidin-4-ones in good yields using microwave irradiation technique on silica as solid support under solvent free conditions. The synthesized compounds were characterized by elemental microanalysis, infrared spectroscopy, ${ }^{1} \mathrm{H}$ NMR, and mass spectroscopy. All the synthesized thiazolidinones were investigated for their antimicrobial and antifungal activities. The results of the biological activities revealed that the compounds $\mathbf{3 b}, \mathbf{3 d}, \mathbf{3} \mathbf{f}$ and $\mathbf{3 h}$ exhibited excellent antibacterial activities while $\mathbf{3 d}$ and $\mathbf{3 h}$ exhibited good antifungal activity.
\end{abstract}

Key Words: Quinoxalines, Silica, Microwave irradiation, Antibacterial, Antifungal

\section{Introduction}

Quinoxaline derivatives have been found to be biologically active compounds having antibacterial, antifungal, antiinflammatory, antidepressant, anthelmintic and herbicidal properties. ${ }^{1-3}$ They also have antimicrobial, ${ }^{4}$ antimycobacterial, ${ }^{5}$ anticancer ${ }^{6}$ and antiallergic properties. ${ }^{7}$ In view of the above interest in these compounds and in continuation of our studies on the cyclization of heterocyclic compounds ${ }^{8-10}$ we have investigated arenecarbaldehyde 3-methylquinoxalin-2-yl-hydrazones in ring-closure reactions with thioglycolic acid under microwave irradiation technique under solvent free conditions on silica support. Microwave-induced Organic Reaction Enhancement $[\mathrm{MORE}]$ chemistry ${ }^{11}$ has received considerable attention in the recent years due to several advantages like short reaction time, ease of work-up, excellent yields and due to its cost effectiveness (only simple glassware needed). Moreover, it is an environmentally friendly technique and is understood to be a step towards green chemistry. ${ }^{12}$ Most of the reactions in microwave oven are carried out in solution phase. However, in these solution-phase reactions, the development of high pressure, which leads to explosion, and the use of sealed containers are some of the limitations. During recent years, a novel practical dimension to the microwave heating protocols has been added by accomplishing reactions on solid supports under solvent-free conditions. A reaction can be carried out by adsorbing the reactants on an inorganic solid support viz., alumina, silica, clay and zeolite in a sealed or open vessel under microwave environment. Though reactants are adsorbed onto the solid support and exposed to microwaves only the reactants absorb the radiations. The solid support does not absorb or restrict their transmission. These solvent-free microwave assisted reactions provide an opportunity to work with open vessels thus avoiding the risk of high-pressure development and increasing the potential of such reactions to upscale. ${ }^{12}$ Usage of solid mineral supports during microwave irradiation technique has several advantages. When reactants are adsorbed onto solid supports, good dispersion of active (reagent) site leads to significant improvement of reactivity due to availability of large surface area and the constraints of the (molecular dimensions) pores and the characteristics of the surface adsorption will also lead to useful improvement in reaction selectivity. ${ }^{12}$ Mineral supports behave as efficient absorbers of microwave energy with consequently attaining more homogeneity in temperature when compared to carrying out reactions in solution phase. There are distinct advantages of these solvent-free protocols since they provide reduction or elimination of solvents thereby preventing pollution in organic synthesis 'at source'. ${ }^{12}$ Thiazolidinones, synthesized via variety of routes such as reaction between benzylidene-amines and mercaptoacetic acid ${ }^{13}$ or by condensation of either aliphatic or aromatic moieties containing a formyl group with different aminothiols ${ }^{14}$ or by refluxing a solution of arylhydrazones and thioglycolic acid in DMF in the presence of anhydrous $\mathrm{ZnCl}_{2}{ }^{15}$ etc., ${ }^{16-17}$ are known to have antibacterial, antifungal, anticonvulsant, anti-inflammatory, anti-diarrhoeal, anti-HIV, anticancer, antimicrobial properties ${ }^{18-24}$ and are also found to be new therapeutic agents for Type-2 Diabetes. ${ }^{25}$ Keeping in view the importance of microwave assisted organic synthesis under solvent free conditions and biological activity of thiazolidinones, we here in report, rapid and efficient conversion of arenecarbaldehyde-3-methylquinoxalin-2-yl-hydrazones (2) to the corresponding heterocyclic thiazolidin-4-ones (3) (Scheme 1) using microwave assisted synthesis on silica support under solvent free conditions. The synthesized compounds were screened for antibacterial and antifungal activity.

\section{Experimental}

Melting points were determined in open capillaries using Buchi 530 apparatus and are uncorrected. The purity of the com- 

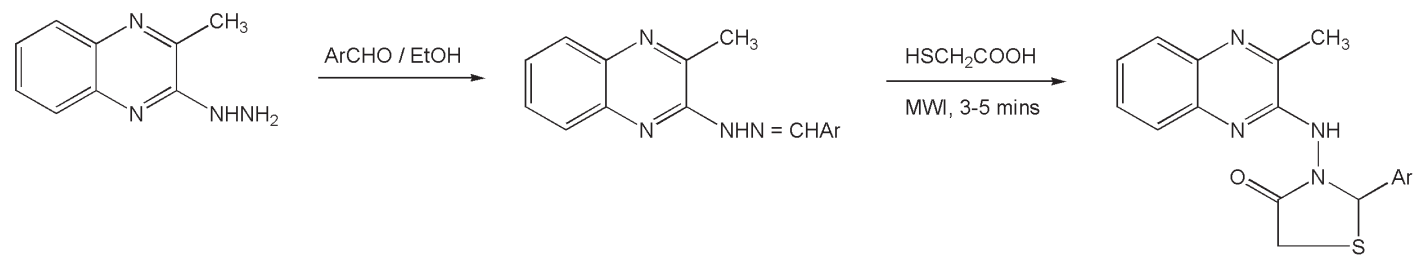

Scheme 1. Design of target compounds (3a-h)

Table 1. Physical constants of compounds $3 \mathbf{a}-\mathbf{h}^{a}$

\begin{tabular}{cccccc}
\hline Entry & $\mathrm{Ar}$ & Reaction Period (mins) & Yield (\%) & M.P. $\left({ }^{\circ} \mathrm{C}\right)$ & Mol. formula (Mol. Wt.) \\
\hline 3a & $\mathrm{C}_{6} \mathrm{H}_{5}$ & 4.0 & 75 & $130-131$ & $\mathrm{C}_{18} \mathrm{H}_{16} \mathrm{~N}_{4} \mathrm{OS}(336)$ \\
3b & $m-\mathrm{NO}_{2}-\mathrm{C}_{6} \mathrm{H}_{4}$ & 3.5 & 79 & $153-155$ & $\mathrm{C}_{18} \mathrm{H}_{15} \mathrm{~N}_{5} \mathrm{O}_{3} \mathrm{~S}(381)$ \\
3c & $p-\mathrm{NO}_{2}-\mathrm{C}_{6} \mathrm{H}_{4}$ & 3.0 & 81 & $194-196$ & $\mathrm{C}_{18} \mathrm{H}_{15} \mathrm{~N}_{5} \mathrm{O}_{3} \mathrm{~S}(381)$ \\
3d & $p-\mathrm{CH}_{3}-\mathrm{C}_{6} \mathrm{H}_{4}$ & 5.0 & 88 & $174-176$ & $\mathrm{C}_{19} \mathrm{H}_{18} \mathrm{~N}_{4} \mathrm{OS}(350)$ \\
3e & $p-\mathrm{OCH}_{3}-\mathrm{C}_{6} \mathrm{H}_{4}$ & 4.5 & 83 & $173-175$ & $\mathrm{C}_{19} \mathrm{H}_{18} \mathrm{~N}_{4} \mathrm{O}_{2} \mathrm{~S}(366)$ \\
3f & $o-\mathrm{OH}-\mathrm{C}_{6} \mathrm{H}_{4}$ & 4.5 & 75 & $145-146$ & $\mathrm{C}_{18} \mathrm{H}_{16} \mathrm{~N}_{4} \mathrm{O}_{2} \mathrm{~S}(352)$ \\
3g & $p-\mathrm{Cl}_{6}-\mathrm{C}_{6} \mathrm{H}_{4}$ & 4.0 & 71 & $128-130$ & $\mathrm{C}_{18} \mathrm{H}_{15} \mathrm{ClN} \mathrm{N}_{4} \mathrm{OS}(370.5)$ \\
3h & $p-\mathrm{OH}-m-\mathrm{OCH} \mathrm{H}_{3}-\mathrm{C}_{6} \mathrm{H}_{3}$ & 5.0 & 70 & $210-211$ & $\mathrm{C}_{19} \mathrm{H}_{18} \mathrm{~N}_{4} \mathrm{O}_{3} \mathrm{~S}(382)$ \\
\hline
\end{tabular}

${ }^{a}$ All new compounds showed $\mathrm{C}, \mathrm{H}, \mathrm{N}$ analysis within $\pm 0.4 \%$.

pounds was checked by ascending TLC on Merck's precoated silica-gel plates $(0.25 \mathrm{~mm})$. Microwave irradiations were carried out in microwave oven specially designed for organic synthesis (LG Electronics, model MG-605AP, 2450 MHZ, 1000 W). Infra red (IR) spectra were recorded in $\mathrm{KBr}$ pellets on Schimadzu IR Prestige-21 FT-IR spectrophotometer $\left(v_{\max }\right.$ in $\left.\mathrm{cm}^{-1}\right) ;{ }^{1} \mathrm{H}$ NMR spectra on Bruker DRX300 spectrometer using TMS as internal standard (chemical shifts in $\delta, \mathrm{ppm}$ ) in DMSO- $d_{6}$; elemental analysis on a Carla Erba 1108 elemental analyzer and mass spectra on a VG-70-S mass spectrometer. 2-Hydrazino-3-methylquinoxaline (1) was prepared by reaction of 2-chloro-3methyl quinoxaline with hydrazine hydrate as per the literature protocol. ${ }^{26}$ Arene carbaldehyde-3-methylquinoxalin-2-yl hydrazones (2a-h) were prepared according to the general procedure. $^{9,27}$

General procedure. A mixture of appropriate heterocyclic hydrazone 2 ( $2 \mathrm{mM})$ and thioglycolic acid $(2 \mathrm{mM})$ was mixed in pestle and mortar homogeneously along with $1 \mathrm{~g}$ of silica gel (60 - 120 mesh size) for about a period of three minutes. This mixture is subjected to microwave irradiation at $60 \%$ power output (540 watt) for the period indicated in Table 1. After completion of the reaction (monitored by TLC), the reaction mixture was cooled to room temperature and triturated with water and extracted using $3 \times 20 \mathrm{~mL}$ portions of ethyl acetate, dried over anhydrous sodium sulpahte and concentrated in vacuo to afford 3 . All the synthesized compounds were recrystallized from ethanol. The yields obtained along with the melting points of final compounds are tabulated in Table 1.

3-(2-Methylquinoxalin-3-yl)-2-phenylthiazolidin-4-one (3a): Light-yellow solid; IR (KBr) $v_{\max } 3037,3019,2979,2943$, 2901, 1708, 1596, 1504, 1205, 1167. ${ }^{1} \mathrm{H} \mathrm{NMR}\left(\mathrm{CDCl}_{3}\right) \delta 8.01-$ $8.12(\mathrm{~d}, 2 \mathrm{H}, J=8.1 \mathrm{~Hz}) ; 7.67-7.75(\mathrm{~d}, 2 \mathrm{H}, J=8.1 \mathrm{~Hz}) ; 7.06-$ $7.14(\mathrm{~m}, 5 \mathrm{H}) ; 5.92(\mathrm{~s}, 1 \mathrm{H}, \mathrm{CH}) ; 3.27-3.38\left(\mathrm{~s}, 2 \mathrm{H}, \mathrm{CH}_{2}\right) ; 2.32$ (s, $3 \mathrm{H}, \mathrm{CH}_{3}$ ). HRMS (FAB) Calcd. for $\mathrm{C}_{18} \mathrm{H}_{16} \mathrm{~N}_{4} \mathrm{OS} 336.4167$, Found 336.4163.
3-(2-Methylquinoxalin-3-yl)-2-(3-nitrophenyl)thiazolidin4-one (3b): Light-yellow solid; IR (KBr) $v_{\max } 3050,3025$, 2975, 2945, 2907, 1712, 1612, 1510, 1557, 1364, 1200, 1170. ${ }^{1} \mathrm{H}$ NMR $\delta$ 8.11-8.16 (d, 2H, $\mathrm{H}_{2}{ }^{\prime} \& \mathrm{H}_{4}$ ' Ph); 8.03-8.05 (d, 2H, $J=8.3 \mathrm{~Hz}) ; 7.66-7.73$ (d, 2H, $J=8.3 \mathrm{~Hz}) ; 7.34-7.43$ (d, 2H, $\mathrm{H}_{5}{ }^{\prime} \& \mathrm{H}_{6}$ ' $\mathrm{Ph}$ ); 5.92 (s, 1H, CH); 3.29-3.36 (s, 2H, $\left.\mathrm{CH}_{2}\right) ; 2.36$ (s, 3H, $\mathrm{CH}_{3}$ ). HRMS (FAB) Calcd. for $\mathrm{C}_{18} \mathrm{H}_{15} \mathrm{~N}_{5} \mathrm{O}_{3} \mathrm{~S} 381.0948$, Found 381.0946.

3-(2-Methylquinoxalin-3-yl)-2-(4-nitrophenyl)thiazolidin4-one (3c): Light-yellow solid; IR (KBr) $v_{\max } 3030,3015$, 2960, 2945, 2900, 1705, 1605, 1500, 1562, 1362, 1203, 1169. ${ }^{1} \mathrm{H}$ NMR $\delta$ 8.06-8.12 (d, 2H, H3' \& $\left.\mathrm{H}_{5}{ }^{\prime} \mathrm{Ph}\right) ; 7.97-8.03(\mathrm{~d}, 2 \mathrm{H}$, $J=8.1 \mathrm{~Hz})$; 7.28-7.74 (m, 4H, $J=8.1 \mathrm{~Hz}) ; 7.27-7.39$ (d, 2H, $\left.\mathrm{H}_{2}{ }^{\prime} \& \mathrm{H}_{6}{ }^{\prime} \mathrm{Ph}\right) ; 5.94$ (s, 1H, CH); 3.28-3.38 (s, 2H, $\left.\mathrm{CH}_{2}\right) ; 2.35$ (s, $3 \mathrm{H}, \mathrm{CH}_{3}$ ). HRMS (FAB) Calcd. for $\mathrm{C}_{18} \mathrm{H}_{15} \mathrm{~N}_{5} \mathrm{O}_{3} \mathrm{~S} 381.0956$, Found: 381.0954.

3-(2-Methylquinoxalin-3-yl)-2-(4-methylphenyl)thiazolidin4-one (3d): Yellow solid; IR (KBr) $v_{\max } 3035,3020,2975$, 2940, 2890, 1704, 1606, 1514, 1204, 1170. 'H NMR $\delta$ 7.998.13 (d, 2H, $J=8.1 \mathrm{~Hz})$; 7.54-7.69 (d, 2H, $J=8.1 \mathrm{~Hz})$; 6.78$6.94(\mathrm{~m}, 4 \mathrm{H}) ; 5.92(\mathrm{~s}, 1 \mathrm{H}, \mathrm{CH}) ; 3.29-3.36\left(\mathrm{~s}, 2 \mathrm{H}, \mathrm{CH}_{2}\right) ; 2.62$ (s, 3H, 4' $\left.\mathrm{CH}_{3}\right) ; 2.32$ (s, 3H, $\left.\mathrm{CH}_{3}\right)$. HRMS (FAB) Calcd. for $\mathrm{C}_{19} \mathrm{H}_{18} \mathrm{~N}_{4} \mathrm{OS} 350.4465$, Found 350.4464 .

2-(4-Methoxyphenyl)-3-(2-methylquinoxalin-3-yl)-thiazolidin-4-one (3e): Light-yellow solid; IR (KBr) v $v_{\max } 3045,3016$, 2977, 2945, 2921, 1700, 1602, 1512, 1200, 1165. ${ }^{1} \mathrm{H}$ NMR $\delta$ $7.92-8.12(\mathrm{~d}, 2 \mathrm{H}, J=8.1 \mathrm{~Hz}) ; 7.60-7.81(\mathrm{~d}, 2 \mathrm{H}, J=8.1 \mathrm{~Hz})$; 6.40-7.01 (m, 4H); 5.90 (s, 1H, CH); 3.74 (s, 3H, $\left.\mathrm{OCH}_{3}\right)$; 3.293.34 (s, 2H, $\left.\mathrm{CH}_{2}\right) ; 2.38$ (s, 3H, $\left.\mathrm{CH}_{3}\right)$. HRMS (FAB) Calcd. for $\mathrm{C}_{19} \mathrm{H}_{18} \mathrm{~N}_{4} \mathrm{O}_{2} \mathrm{~S} 366.4462$, Found 366.4460.

2-(2-Hydroxyphenyl)-3-(2-methylquinoxalin-3-yl)-thiazolidin-4-one (3f): Light-yellow solid; IR (KBr) $v_{\max }$ 3420-3550, 3034, 3012, 2965, 2943, 2900, 1704, 1602, 1512, 1205, 1165. ${ }^{1} \mathrm{H}$ NMR $\delta 8.04-8.10(\mathrm{~d}, 2 \mathrm{H}, J=7.9 \mathrm{~Hz}) ; 7.66-7.91(\mathrm{~d}, 2 \mathrm{H}, J=$ 7.9 Hz); 6.61-6.91 (m, 4H, Ph); 5.92 (s, 1H, CH); 5.36 (s, 1H, 
$\mathrm{OH}, \mathrm{D}_{2} \mathrm{O}$ exchangeable); 3.29-3.36 (s, 2H, $\left.\mathrm{CH}_{2}\right) ; 2.33$ (s, 3H, $\mathrm{CH}_{3}$ ). HRMS (FAB) Calcd. for $\mathrm{C}_{18} \mathrm{H}_{16} \mathrm{~N}_{4} \mathrm{O}_{2} \mathrm{~S} 352.4167$, Found 352.4163 .

2-(4-Chlorophenyl)-3-(2-methylquinoxalin-3-yl)-thiazolidin-4-one (3g): Light-yellow solid; IR (KBr) $v_{\max } 3032,3017$, 2976, 2940, 2895, 1702, 1599, 1505, 1202, 1169, 720. ${ }^{1} \mathrm{H}$ NMR $\delta 8.02-8.11(\mathrm{~d}, 2 \mathrm{H}, J=8.1 \mathrm{~Hz}) ; 7.67-7.81(\mathrm{~d}, 2 \mathrm{H}, J=8.1 \mathrm{~Hz})$; 7.01-7.24 (m, 4H); 5.91 (s, 1H, CH); 3.29-3.36 (s, 2H, $\left.\mathrm{CH}_{2}\right)$; 2.34 (s, 3H, $\mathrm{CH}_{3}$ ). HRMS (FAB) Calcd. for $\mathrm{C}_{18} \mathrm{H}_{15} \mathrm{ClN}_{4} \mathrm{OS}$ 370.8658 , Found 370.8655 .

2-(4-Hydrox-3-methoxyyphenyl)-3-(2-methylquinoxalin3-yl)-thiazolidin-4-one (3h): Yellow solid; IR (KBr) $v_{\max } 3440$ 3525, 3037, 3019, 2979, 2943, 2901, 1706, 1592, 1504, 1204, 1170. ${ }^{1} \mathrm{H}$ NMR $\delta 8.06-8.22(\mathrm{~d}, 2 \mathrm{H}, J=8.2 \mathrm{~Hz}) ; 7.63-7.71(\mathrm{~d}$, $2 \mathrm{H}, J=8.2 \mathrm{~Hz}$ ); 6.38-6.54 (m, 3H, Ph); 5.92 (s, 1H, CH); 5.12 (s, $1 \mathrm{H}, \mathrm{OH}, \mathrm{D}_{2} \mathrm{O}$ exchangeable); $3.73\left(\mathrm{~s}, 3 \mathrm{H}, \mathrm{OCH}_{3}\right) ; 3.27-3.33$ $\left(\mathrm{s}, 2 \mathrm{H}, \mathrm{CH}_{2}\right) ; 2.29\left(\mathrm{~s}, 3 \mathrm{H}, \mathrm{CH}_{3}\right)$. HRMS (FAB) Calcd. for $\mathrm{C}_{19}$ $\mathrm{H}_{18} \mathrm{~N}_{4} \mathrm{O}_{3} \mathrm{~S} 382.4459$, Found 382.4452 .

Antimicrobial activity. The test microorganisms were obtained from Department of Microbiology, Madurai Medical College and Research Institute, Madurai, India. Muller Hinton agar plates $\left(37^{\circ} \mathrm{C}, 24 \mathrm{~h}\right)$ and Sabouraud's dextrose agar plates $\left(26^{\circ} \mathrm{C}, 48-72 \mathrm{~h}\right)$ were used for the cultivation of bacteria and fungi, respectively. The zone of inhibition was measured in $\mathrm{mm}$. All synthesized compounds were screened for antibacterial activity by cup-plate agar diffusion method ${ }^{28}$ against gram-positive species Staphylococcus aureu, and Bacillus subtilis and gram-negative species Escherichia coli, Pseudomonas aerogenosa and Klebsilla aerogenes in the concentration of $25 \mu \mathrm{g} /$ $\mathrm{mL}$. These compounds were also screened for antifungal activity against fungi Aspergillus niger, Aspergillus flavus and Candida albican by the paper disc diffusion method ${ }^{29}$ in the same concentration. The activities were compared with standard drugs Ciprofloxacin, Norfloxacin, Salicylic acid and Clotrimazole. All the synthesized compounds were dissolved in DMF, which was used as a control.

\section{Results and Discussion}

Chemical synthesis. We report herein synthesis of quinoxalinyl thiazolidinones using microwave irradiation technique on silica support under solvent free conditions. Substituted hydrazones and thioglycolic acid were adsorbed onto silica gel and irradiated in a microwave oven under solvent free conditions for about 3 - 5 minutes at 540 watt power output to afford thiazolidinones. When the reaction mixture was subjected to microwave irradiation at either $<540$ watts or $>540$ watts, yields obtained were very poor. Probably at higher power output thioglycolic acid is evaporating (boiling point: $101.5^{\circ} \mathrm{C}$ ) and thereby the reaction progress is hampered giving poor yields. All the microwave conversions were carried out in triplicates to examine the reproducibility. The starting material, 2-hydrazino3-methylquinoxaline (1), was prepared as per the literature protocol. $^{26}$

All the synthesized compounds (3a-h) were characterized by elemental analysis (CHN) and spectral (IR, ${ }^{1} \mathrm{H}$ NMR and MS) data. Formation of hydrazones (2a-h) was confirmed by previously reported melting points ${ }^{9}$ and the absence of primary amino group at $3350 \mathrm{~cm}^{-1}$ in IR spectra. An important characteristic feature in the ${ }^{1} \mathrm{H}$ NMR spectra of $\mathbf{3}$ was the disappearance of the signals at $\delta 8.5$ and 9.3 for aldehydic $\mathrm{H}$ and for $\mathrm{NH}$, respectively, which were present in the spectra of the intermediate hydrazones (2a-h). IR spectral analysis of the final compounds (3a-h) show absorption band at $\sim 1700 \mathrm{~cm}^{-1}, \sim 1200 \mathrm{~cm}^{-1}$ and $\sim 1170 \mathrm{~cm}^{-1}$ due to $\mathrm{C}=\mathrm{O}, \mathrm{C}-\mathrm{S}$ and $\mathrm{C}-\mathrm{N}$ functions of thiazolidinone moiety respectively. In ${ }^{1} \mathrm{H}$ NMR spectra, peak at $\delta$ 7.66-8.33 and $\delta 6.35-8.07$ indicates the presence of quinoxaline ring and phenyl ring respectively. Elemental $(\mathrm{CHN})$ analysis indicated that calculated and observed values were within the acceptable limits $( \pm 0.4 \%)$.

Antibacterial activity. All the compounds were active against Escherichia coli and Staphylococcus aureus. Compounds $\mathbf{3 b}$, 3e, 3f and $\mathbf{3 h}$ were active against Bacillus subtilis while $\mathbf{3 a}, \mathbf{3 c}$, 3d, 3f and $\mathbf{3 h}$ showed good activity against Pseudomonas aerogenosa. Compounds $\mathbf{3 b}, \mathbf{3 d}$ and $\mathbf{3 g}$ were active against Klebsilla aerogenes. The other compounds showed either moderate or less activity against these organisms.

Antifungal activity. Most of the synthesized compounds were found to possess moderate activity against tested fungi. Compounds $\mathbf{3 d}$ and $\mathbf{3 h}$ were found to be most active against Aspergillus flavus and Candida albican respectively. The antifungal activities of test compounds were compared with standard Salicylic acid $(20-30 \mathrm{~mm})$ and Clotrimazole $(25-30 \mathrm{~mm})$. The results of antibacterial and antifungal activity tests are summarized in Tables 2 and 3.

\section{Conclusions}

In conclusion, a microwave-assisted rapid synthesis of thiazolidinones has been achieved under solvent free conditions. While this environmentally friendly method does not differ significantly from the conventional method in terms of product nature and yield, ${ }^{30}$ it however provides advantages, such as shorter reaction time, solvent-free conditions and minimal purification of the products. All the synthesized compounds were screened for antibacterial activity against Escherichia coli, Bacillus subtilis, Pseudomonas aerogenosa, Klebsilla aerogenes and Staphylococcus aureus and compound $\mathbf{3 b}$ was found

Table 2. Antibacterial ${ }^{a}$ activity of thiazolidinone derivatives

\begin{tabular}{cccccc}
\hline Entry & EC & SA & BS & PA & KA \\
\hline $\mathbf{3 a}$ & ++ & +++ & - & ++++ & - \\
$\mathbf{3 b}$ & + & ++ & +++ & + & ++++ \\
$\mathbf{3 c}$ & ++ & +++ & - & +++ & - \\
$\mathbf{3 d}$ & +++ & + & - & ++ & ++++ \\
$\mathbf{3 e}$ & ++ & ++ & +++ & - & - \\
$\mathbf{3 f}$ & + & +++ & ++++ & +++ & - \\
$\mathbf{3 g}$ & +++ & ++ & - & - & +++ \\
$\mathbf{3 h}$ & ++ & ++ & ++ & +++ & - \\
Norfloxacin & ++++ & ++++ & ++++ & ++++ & ++++ \\
Ciprofloxacin & ++++ & +++ & ++++ & ++++ & ++++ \\
\hline
\end{tabular}

${ }^{a}$ Data are zones of inhibition (mm). EC is Escherichia coli; SA is Staphylococcus aureus; $\mathrm{BS}$ is Bacillus subtilis; PA is Pseudomonas aerogenosa and KA is Klebsilla aerogenes. ${ }^{-}=<10 \mathrm{~mm} ;+=10-15 \mathrm{~mm} ;++=15-$ $20 \mathrm{~mm} ;+++=20-25 \mathrm{~mm} ;++++=25-30$. 
Table 3. Antifungal ${ }^{a}$ activity of thiazolidinone derivatives

\begin{tabular}{cccc}
\hline Entry & $\begin{array}{c}\text { Inhibition of } \\
\text { A. niger }\end{array}$ & $\begin{array}{c}\text { Inhibition of } \\
\text { A. flavus }\end{array}$ & $\begin{array}{c}\text { Inhibition of } \\
\text { C. albican }\end{array}$ \\
\hline 3a & + & - & + \\
3b & ++ & ++ & + \\
3c & ++ & + & - \\
3d & - & ++++ & ++ \\
3e & ++ & - & - \\
3f & + & - & + \\
3g & ++ & ++ & ++ \\
3h & +++ & ++ & ++++ \\
Salicylic acid & ++++ & +++ & ++++ \\
Clotrimazole & ++++ & ++++ & ++++ \\
DMF & - & - & -
\end{tabular}

${ }^{a}$ Data are zones of inhibition $(\mathrm{mm}) .^{-}=<10 \mathrm{~mm} ;+=10-15 \mathrm{~mm} ;++=$ $15-20 \mathrm{~mm} ;+++=20-=20-25 \mathrm{~mm} ;++++=25-30 \mathrm{~mm}$.

to be the most active compound. Thiazolidinones were also screened for antifungal activity against Aspergillus niger, Aspergillus flavus and Candida albican and $\mathbf{3 d}$ and $\mathbf{3 h}$ were the most active compounds.

Acknowledgments. We thank UGC, India for financial support and the SAIF, CDRI, Lucknow, India and the SAIF, Panjab University, Chandigarh, India for analytical and spectral data.

\section{References}

1. Kurasawa, Y.; Takad, A. Heterocycles 1986, 24, 2321.

2. Kurasawa, Y.; Muramatsu, M.; Yamazaki, K.; Tajima, S.; Okamoto, Y.; Takada, A. J. Heterocycl. Chem. 1986, 23, 1379.

3. Skata, G.; Makino, K.; Kurasawa, Y. Heterocycles 1988, $27,2481$.

4. Metzner, J.; Lippmann, E.; Weber, F .G.; Westphal, G. Pharmazie 1981, 36, 368.

5. Shinde, B. R.; Pai, N. R. Indian Drugs 1989, 27, 32.

6. Boido, A.; Vazzana, I.; Sparatore, F. I L Farmaco 1994, 49, 97.
7. Love, B.; Musser, J. H.; Brown, R. E.; Jones, H.; Kahen, R.; Huang, F. C.; Khandwala, A.; Sonnino, G. P.; Leibowitz, M. J. Med. Chem. 1985, 28, 363 .

8. Rao, V. S.; Chandra Sekhar, K. V. G. Synth. Comm. 2004, 34, 2153.

9. Kumar, D.; Chandra Sekhar, K. V. G.; Dhillon, H.; Rao, V. S.; Varma, R. S. Green Chem. 2004, 6, 156.

10. Chandra Sekhar, K. V. G.; Rao, V. S.; Murali Krishna Kumar, M. J. Enzym. Inhib. Med. Ch. 2009, 24, 871.

11. Banik, B. K.; Manhas, M. S.; Newas, S. N. ; Bose, A. K. Bioorg. Med. Chem. Lett. 1993, 33, 2363.

12. Varma, R. S. Green Chem. 1999, 1, 43.

13. Bolognese, A.; Correale, G.; Manfra, M.; Lavecchia, A.; Novellino, E.; Barone, V. Org. Biomol. Chem. 2004, 2, 2809.

14. Sattigeri, V. J.; Soni, A.; Singhal, S.; Khan, S.; Pandya, M.; Bhateja, P.; Mathur, T.; Rattan, A.; Khanna, J.; Mehta, A. Arkovic 2005, ii, 46 .

15. Cacic, M.; Molnar, M.; Balic, T.; Rajkovic, V. Molecules 2009, 14, 2501.

16. Singh, S. P.; Parmar, S. S.; Raman, K.; Stenberg, V. I. Chem. Rev. 1981, 81, 175.

17. Hamama, W. S.; Ismail, M. A.; Shaaban, S.; Zoorob, H. H. J. Heterocyclic Chem. 2008, 45, 939.

18. Garnaik, B. K.; Behera, R. K. Indian J. Chem. 1988, $27 B, 1157$.

19. Vladzimirskaya, V.; Novikevich, T.; Demchuk, G. Farm. Zh. (Kiev) 1991, 6, 67.

20. Lakhan, R.; Singh, R. L. J. Agric. Food Chem. 1991, 39, 580.

21. Pawar, R. P.; Andurkar, M.; Vibhute, Y. B. J. Indian Chem. Soc. 1999, 76, 271.

22. El-Feky, S. A. H. Pharmazie 1993, 48, 894.

23. Grasso, S.; Chimirri, A.; Zappala, M. Farmaco 1986, 41, 713.

24. Samir, B.; Wesam, K.; Ahmed, A. F. Eur. J. Med. Chem. 2007, 42, 948 .

25. Baihua. US Pat 6821991, 2004.

26. Shiho, D.; Tagami, S. J. Am. Chem. Soc. 1960, 82, 4044.

27. Barry, A. L. The Antimicrobial Susceptibility Test, Principle and Practices, 4th ed.; ELBS: London, 1999; p 180.

28. Seelay, H. W.; Denmark, P. J. V. Microbes in action; W. H. Freeman \& Co: New York, 1972.

29. Vyas, D. A.; Chauhan, N. A.; Parikh, A. R. Indian J. Chem. 2007, $46 B, 1699$.

30. Prakash, O.; Bhardwaj, V.; Kumar, R.; Tyagi, P.; Aneja, K. R. Eur. J. Med. Chem. 2004, 39, 1073. 